COMMENT. IPEAF is a benign form of temporal lobe epilepsy without structural brain lesion or known cause. The authors attach the term 'idiopathic' because they consider some unrecognized genetic factor might play a role in etiology. The authors are to be commended on their historical review of auditory seizures that includes the early references of Holmes (1927), Lennox (1960), Penfield (1951), and Currie (1971). Interest in auditory partial epilepsy has been renewed following the report of a syndrome of autosomal dominant partial epilepsy with auditory features (Ottman et al, 1995).

\title{
EPILEPSY OUTCOME IN PERIVENTRICULAR NODULAR HETEROTOPIA
}

The clinical, electroencephalographic, and neuroimaging features and course of seizures in 16 patients with periventricular (subependymal) nodular heterotopia (PNH) were investigated at the University of Bologna, Italy. The mean follow-up was 17.3 years, mean age at follow-up 29 years (range 15-48 years). Simple cases of PNH ( 8 cases; 5 women and 3 men) were characterized by normal intelligence and seizures that were usually partial, associated with focal EEG, began in the second decade (mean age at seizure onset 19.2 years (range 17-22), were infrequent and tended to disappear. The 8 complicated PNH plus cases ( 3 women and 5 men) were distinguished by mental retardation and seizures that began in the first decade (mean 4.25 years; range 1-14), associated with focal and bisynchronous EEG abnormalities, were very frequent and multiple in pattern (partial, secondarily generalized, akinetic or tonic clonic), and often medically refractory. The MRI in simple PNH cases showed only periventricular nodules, whereas PNH plus cases were characterized by other brain malformations, including subcortical heterotopia, polymicrogyria, focal dysplasia, schizencephaly, cortical infolding, agenesis of the corpus callosum, mega cisterna magna and cerebellar atrophy. (d'Orsi G, Tinuper P, Bisulli F, et al. Clinical features and long term outcome of epilepsy in periventricular nodular heterotopia. Simple compared with plus forms. J Neurol Neurosurg Psychiatry 2004;75:873-878). (Respond: Professor P Tinuper, Department of Neurological Sciences, University of Bologna, Via Ugo Foscolo 7, 40123 Bologna, Italy).

COMMENT. Patients with seizures associated with periventricular nodular heterotopia (PNH) are classified in two groups: 1) Simple PNH and 2) PNH plus. The PNH plus cases characterized by additional brain malformations have an earlier presentation and carry a poor prognosis.

Genetics of periventricular heterotopia. Four families of $\mathrm{PNH}$ are reported in which FLN1 mutations caused PNH in men. In 2 families mild missense mutations were transmitted from mother to son and from a father to daughter, causing mild phenotypes, with unilateral PNH in one. In a third family, an affected man with classic PNH (bilateral PNH, enlarged cisterna magna, and cerebellar vermis hypoplasia) had mosaic mutations of FLN1 gene, and in the fourth family, a truncating mutation was associated with early postnatal death of a boy with $\mathrm{PNH}$, focal polymicrogyria, and cardiovascular and genitourinary malformations. X-linked PNH caused by FLN1 mutations in men is clinically heterogeneous and caused by different genetic mechanisms. (Guerrini R, et al. Neurology July (1 of 2) 2004;63:51-56). Affected women usually have epilepsy and normal or borderline intelligence. 